\title{
Using Health Helpline Mediated Self-Swabbing as a Surveillance Tool for Influenza
}

\author{
Danielle McGolrick ${ }^{\star 1}$, Paul Belanger ${ }^{2}$, Allison Maier ${ }^{2}$, Harriet Richardson ${ }^{1}$, Kieran \\ Moore $^{2}$, Nino Lombardi ${ }^{3}$ and Anna Majury, ${ }^{1,3}$
}

${ }^{1}$ Queen's University, Kingston, ON, Canada; ${ }^{2}$ Kingston, Frontenac, and Lennox \& Addington Public Health Unit, Kingston, ON, Canada; ${ }^{3}$ Public Health Ontario Laboratories, Toronto \& Kingston, ON, Canada

\section{Objective}

Explore the use and feasibility of self-swabbing mediated by a telephone health helpline (THHL) as a complementary tool for surveillance of influenza and other common respiratory viruses in Ontario, Canada.

\section{Introduction}

Currently, three main sources of data are used to monitor the prevalence of influenza in Ontario: Public Health Agency of Canada's (PHAC) FluWatch, Ontario's Acute Care Enhanced Surveillance (ACES) data and Public Health Ontario's (PHO) traditional laboratory data. However, a limitation of these data sources is that it typically underestimates the burden of infection in populations living in remote communities and/or populations with less severe symptoms. This study describes a self-swabbing surveillance system mediated by a THHL that uses syndromic surveillance tools to recruit and monitor participants with influenza-like illness. The intent of this system is not to replace, but rather to complement other surveillance systems and clinical based testing for influenza, thereby extending the reach of surveillance through the use of self-swabbing. An additional rationale for this type of surveillance system is that it can reduce transmission of infection by limiting the number of visits to emergency departments or doctors' offices, thereby reducing contact with the young and elderly populations, who are at most risk for infection.

\section{Methods}

Recruitment and System Operation: Participants were recruited through a THHL available to all residents of Ontario. Callers were triaged based on assessment by a registered nurse and deemed eligible to partake in the study if classified under the "referral" or "self-care" categories. Participants must also be at least 2 years old, and have one of the following symptoms: fever, cough, sore throat or coryza. Upon agreeing to participate, participant information was collected and a self-swabbing package, including a swabbing kit, consent form, questionnaire, and description of the study with instructions for participation, was sent out the next business day. The participant completed the documentation, used the nasal swab to obtain a specimen, and returned them to the laboratory. Data was then collected and the swab was tested for influenza viruses.

Analyses and evaluation: The questionnaire data and laboratory results were used to evaluate the feasibility of the surveillance system. Evaluation included a descriptive analysis of the population captured by these methods and a basic assessment of the operational aspect of the system. Detection of respiratory viruses using the self-swabbing methodology was reported based on the molecular test results. Furthermore, the timely detection of influenza emerging in Ontario was compared to other surveillance systems that are routinely used in the province via peak comparison methods.

\section{Results}

A total of 2431 participants were successfully sent a package and $666(27.40 \%)$ returned a package with consent, thus their samples were tested. The mean and median number of days between the time of call to the THHL and the time a package was received at the laboratory was approximately 10.4 and 8.6 days, respectively. The time between swab collection and package reception was 4.9 days on average, or a median of 4 days. Given these timelines, selfswabbing proved to be a viable method of detecting influenza and other respiratory viruses as $279(42 \%)$ specimens tested positive for a virus by molecular methods. In terms of early detection, this surveillance system adequately captured the 2014 influenza B season in a timely manner when compared to data generated by FluWatch, ACES, and PHO's traditional laboratory data; however, it did not necessarily detect the emergence of influenza B any earlier than alternative sources. Surveillance of influenza A by our system was also evaluated; however, the number of cases of influenza A peaked approximately two weeks after that reported by PHO's data.

\section{Conclusions}

This study demonstrated the potential a THHL-mediated selfswabbing surveillance tool has for capturing data on a population that is not typically included in existing surveillance methods, and for the timely detection of influenza for surveillance purposes. Certain limitations of this study made for a challenging evaluation of the system. Despite these challenges, this surveillance system was able to obtain viable specimens for laboratory testing and capture seasonal patterns of influenza $\mathrm{B}$ in time with the aforementioned alternative surveillance systems.

\section{Keywords}

influenza; self-swabbing; surveillance; respiratory viruses; health helpline

\section{*Danielle McGolrick \\ E-mail: 8dcm3@queensu.ca}

